\title{
REVISIÓN TEÓRICA DE EVALUACIÓN DEL POTENCIAL DE COLAPSO EN LAS DUNITAS DE MEDELLÍN
}

\author{
Óscar Egidio Rodríguez¹, Eileen Carolina Salgado², Adriana María Gallo², Eduardo Danilo Vanegas² \\ ${ }^{1}$ Ingeniero civil, Especialista en Vías \\ 2 Estudiante de Ingeniería civil \\ Grupo de investigación teRMOMEC, Universidad Cooperativa de Colombia, Medellín, Colombia \\ Correo electrónico: semillerogig@gmail.com
}

Recibido: 28 de noviembre del 2013. Aprobado: 2 de diciembre del 2013.

Cómo citar este artículo: Ó. E. Rodríguez, E. C. Salgado, A. M. Gallo y E. D. Vanegas, "Revisión teórica de evaluación del potencial de colapso en las dunitas de Medellín". Ingeniería Solidaria, vol. 10, n.ำ 17, pp. 193-197, en.-dic., 2014. doi: http://dx.doi.org/10.16925/in.v10i17.882

Resumen. En el siguiente artículo se hace referencia a la primera etapa de una investigación que está enmarcada por una revisión bibliográfica y teórica de todos los criterios, métodos y ensayos que se han utilizado a través del tiempo para determinar el grado de colapsabilidad de los suelos y, posteriormente, se realizará una segunda etapa que consistirá en la aplicación de una serie de ensayos de laboratorio para obtener las conclusiones pertinentes que nos permita identificar el grado de colapso del suelo. Esta investigación se lleva a cabo en la ciudad de Medellín, principalmente en la zona oriental donde se encuentra ubicada la dunita. La idea surgió por una serie de eventos que se han venido presentado en este sector, a lo largo de los años, donde han sido afectadas algunas vías principales como son la autopista Medellín-Bogotá, que comunica a Medellín con la capital de Colombia, la vía Las Palmas que comunica a Medellín con el aeropuerto internacional José María Córdoba.

Palabras clave: dunita, grado de colapsabilidad, criterios de colapsabilidad, ensayos, eventos catastróficos.

\section{Theoretical Review of Evaluation of the Potential for Collapse of las DUNitas (THE DUNites) OF MEDELLín}

Abstract. This article refers to the first stage of research framed by a bibliographic and theoretical review of all criteria, methods and tests that have been used to determine the degree of collapsibility of soils. A second stage will consist of the application of a series of laboratory tests to obtain the relevant conclusions in order to identify the degree of soil collapse. This research is being carried out in the city of Medellín, mainly in the eastern zone where la dunita (the dunite) is located. The idea arose from a series of events in this sector over the years that have affected some of the main roads, such as the Medellín-Bogotá highway, which connects Medellín to the capital of Colombia, and the Las Palmas highway connecting Medellín to José María Córdoba international airport.

Keywords: dune, degree of collapsibility, tests, catastrophic events.

\section{REVISÃo TEÓRICA DE AVALIAÇÃo do POTENCIAL de Colapso nas “dunitas” de Medellín}

Resumo. Neste artigo, faz-se referência à primeira etapa de uma pesquisa que está delimitada por uma revisão bibliográfica e teórica de todos os critérios, métodos e ensaios que foram utilizados pelo tempo para determinar o grau de colapsabilidade dos solos e, posteriormente, será realizada uma segunda etapa que consistirá na aplicação de uma série de ensaios de laboratório para obter as conclusões pertinentes que nos permita identificar o grau de colapso do solo. Esta pesquisa se realiza na cidade de Medellín, principalmente na área leste onde se encontra a "dunita". A ideia surgiu por uma série de eventos que vêm sendo apresentados nesse setor ao longo dos anos os quais têm afetado algumas vias principais como a que comunica Medellín com a capital da Colômbia, a via Las Palmas, que comunica Medellín com o aeroporto internacional José María Córdoba.

Palabras-chave: "dunita", grau de colapsabilidade, critérios de colapsabilidade, ensaios, eventos catastróficos. 


\section{Introducción 1}

Los suelos colapsables se definen como aquellos que experimentan un cambio de volumen debido a varios factores externos como la saturación del suelo, aumento de las cargas, carencia de agua, factores químicos, alto índice de porosidad y baja densidad.

En el presente trabajo se muestran las metodologías y criterios que serán utilizados para evaluar el grado de colapso que tiene la dunita de Medellín, y con esto poder verificar si los eventos catastróficos ocurridos en esta ciudad están relacionados con este problema de suelos colapsables. A su vez, estas indagaciones nos servirán de apoyo para realizar algunos ensayos de laboratorio, con el fin de determinar las propiedades de los suelos residuales derivados de las dunitas y poder obtener algunos resultados que nos permitan identificar y llegar a la conclusión si este material de Medellín tiene relación con las características que presenta un suelo colapsable.

\section{Metodologías y criterios}

A continuación se presentan las principales metodologías y criterios propuestos por diferentes autores, a través del tiempo, para evaluar el grado de colapsabilidad de los suelos.

Barden L. Madedor A. (1969):

- Una estructura abierta, potencialmente inestable, parcialmente saturada.
- Un valor de tensión suficientemente elevado como para producir una condición de equilibrio metaestable.

- Un valor elevado de succión o cementación, que estabilice los contactos inter-granulares y cuya reducción por humedecimiento lleve al colapso.

Zur y Wiseman (1973):

Define como colapso a cualquier disminución rápida de volumen del suelo, producida por el aumento de cualquiera de los siguientes factores:

- Contenido de humedad (w)

- Grado de saturación (SR)

- Tensión media actuante $(\tau)$

- Tensión de corte $(\sigma)$

- Presión de poros (u)

Moll L. (1975):

Los suelos colapsables poseen una estructura altamente porosa, tiene una fábrica de textura abierta, que puede llegar a resistir esfuerzos razonablemente grandes para condiciones de no saturación, pero que presentan una disminución brusca de volumen, debido al colapso de la estructura al ser saturados, aún para condiciones de esfuerzo relativamente baja.

Lisandro A. Capdevila (2006):

Los suelos colapsables son suelos no saturados que experimentan; cuando están sujetos a saturación,
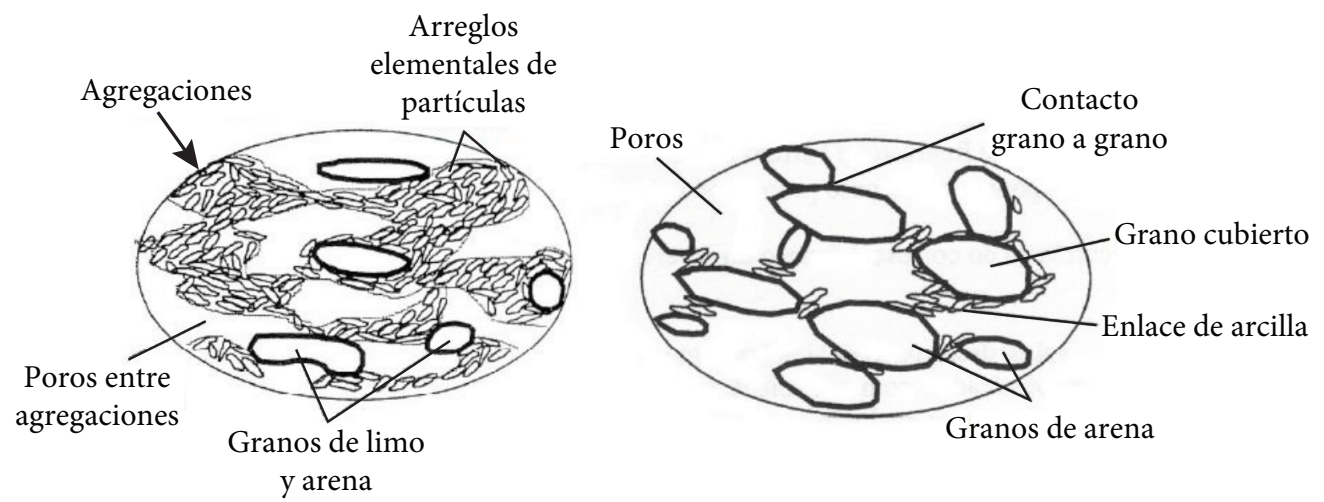

Figura 1. Estructura típica de los suelos colapsables

1 Este artículo se deriva de una ponencia presentada en el I Congreso Nacional de Vías, realizado en noviembre de 2013, en Medellín (Antioquia), Colombia. 
un reacomodo en sus partículas y un excesivo decremento en su volumen con o sin la aplicación de cargas externas.

Cualquier suelo no saturado que no contenga minerales expansivos abundantes puede tener características colapsables.

Características comunes de los suelos colapsables (Reginatto, 1977):
- Estructura macroporosa, con índice de huecos entre relativamente altos a muy altos.

- Granulometría predominante fina, con predominio de limos y de arcilla.

- Estructura mal acomodada, con partículas de mayor tamaño separada por espacios abiertos y unidas entre sí por acumulaciones o «puentes» de material predominante arcillos.

\subsection{Evaluación de la colapsabilidad del suelo}

Tabla 1. Criterios para evaluar la colapsabilidad del suelo

\begin{tabular}{|l|c|l|}
\hline \multicolumn{1}{|c|}{ Criterio propuesto } & Coeficiente de colapsabilidad & \multicolumn{1}{|c|}{$\begin{array}{c}\text { Rango del coeficiente de } \\
\text { colapsabilidad }\end{array}$} \\
\hline Abelev (1948) & $\delta s=\frac{e}{e L+1} \%$ & $6,2>2$ \\
\hline Feda (1960) & $i_{c}=\frac{\left(\frac{m}{S_{t}}\right)-P L}{P l}$ & $0,396<1$ \\
\hline Denisov (1964) & $\frac{e}{e_{L L}}>1$ & $1,2>1$ \\
\hline Clevenger (1985) & $\gamma_{d}=1,38 g / \mathrm{cm}^{3}$ & $1,28<1,38<1,44$ \\
\hline Lin y Wang (1988) & $i_{c z}=\frac{h_{z}-h_{z z}}{h_{1}} * 100$ & $5<7,36<10$ \\
\hline
\end{tabular}

\subsection{Definición de variables}

$\delta s=$ Coeficiente de colapsabilidad

$\Delta e=$ Reducción de la relación de vacíos durante la saturación del suelo

$e L=$ Relación de vacíos antes de la saturación del suelo

$i_{c}=$ Índice de colapsabilidad

$m=$ Contenido natural de agua

$S_{r}=$ Relación de saturación

$P L=$ Límite plástico

$P I=$ Índice de plasticidad

$e=$ Relación de vacíos del suelo natural

$e_{L L}=$ Contenido de agua en el límite líquido

$\Upsilon_{d}=$ Densidad del suelo

$i_{c z}=$ Índice de colapsabilidad del suelo en condición de peso propio

$h_{z}=$ Espesor de la muestra del suelo en la prueba del odómetro respecto a la presión de la sobrecarga en condiciones naturales $h_{z z}=$ Espesor de la muestra del suelo en la prueba del odómetro en condiciones de saturacion

$h_{1}=$ Espesor de la muestra inicial

\section{Ubicación de la dunita de Medellín}

Esta unidad está conformada por un cuerpo principal que aflora al oriente de Medellín. El área es de unos $60 \mathrm{~km}^{2}$. El cuerpo principal se extiende desde la vereda Perico del municipio de Envigado al sur hasta cerca de la cabecera municipal de San Pedro de los Milagros al noroccidente, y está dividido en tres bloques. El bloque meridional está separado del central por la quebrada Santa Elena, donde afloran anfibolitas, y el central está separado del septentrional por los aluviones del río Medellín en el municipio de Bello [2].

Muchas son las hipótesis que se generaron apartir de las multiples eventos de deslizamientos que han ocurrido en la zona oriental de Medellín, afectando al- 
gunas vías importantes de la ciudad como son la autopista Medellín-Bogotá y la vía Las Palmas que conecta a Medellín con el aeropuerto internacional José María Córdoba (JMC); y adicionalmente, a su vez la poblacion también ha sido afectada por estos deslizamientos que dejaron muchas víctimas fatales y damnificados. En la tabla 2 se relacionan algunos eventos representativos ocurridos en esta zona a lo largo del tiempo.

Tabla 2. Relación de eventos

\begin{tabular}{|l|l|c|c|l|}
\hline \multicolumn{1}{|c|}{ Municipio } & \multicolumn{1}{|c|}{ Sector } & Fecha & Víctimas & \multicolumn{1}{c|}{ Eventos } \\
\hline Medellín & Santo Domingo Savio & $29-09-1974$ & 100 & Deslizamiento \\
\hline Medellín & Villatina & $27-09-1987$ & 500 & Deslizamiento \\
\hline Bello & Quebrada El Barro & $06-10-2006$ & 58 & Avenida torrencial \\
\hline Medellín & La Cruz & $31-05-2007$ & 7 & Deslizamiento \\
\hline Medellín & Santo Domingo, La Balastrera & $17-07-2008$ & 4 & Movimiento en masa \\
\hline Medellín & El Poblado, Alto Verde & $16-11-2008$ & 12 & Movimiento en masa \\
\hline Medellín & La Sierra & $13-11-2010$ & 14 & Derrumbe \\
\hline Bello & La Gabriela & $07-12-2010$ & 35 & Movimiento en masa \\
\hline
\end{tabular}

\section{Ensayos de laboratorio}

Los ensayos de laboratorio son los más importantes porque por medio de ellos determinaremos el grado de colapsabilidad que presenta este tipo de material. En la tabla 3 se presentan los ensayos que se realizarán a las muestras, las cuales se tomarán lo más cerca posible donde ocurrieron los eventos anteriormente descritos.

Tabla 3. Ensayos de laboratorio

\begin{tabular}{|l|l|}
\hline \multicolumn{1}{|c|}{ Muestras alteradas } & \multicolumn{1}{c|}{ Muestras inalteradas } \\
\hline Humedad natural & $\begin{array}{l}\text { Propiedades volumétricas y } \\
\text { gravimétricas }\end{array}$ \\
\hline Gravedad específica & Ensayos de consolidación \\
\hline Límites de consistencia & Permeabilidad de los materiales \\
\hline $\begin{array}{l}\text { Granulometría por malla, por } \\
\text { hidrómetro y lavado por la malla } \\
\text { \# 200 }\end{array}$ & \\
\hline $\begin{array}{l}\text { Clasificación de los materiales } \\
\text { por el Sistema Unificado de } \\
\text { Clasificación de Suelos (USCS) }\end{array}$ & \\
\hline
\end{tabular}

Fuente: Elaborado por el grupo de Investigación TERMOMEC

\section{Conclusión}

Es importante resaltar que todos estos criterios son pertinentes y eficaces para determinar el grado de colapsabilidad de la dunita de Medellín, teniendo presente todos los requisitos o normas a la hora de tomar las muestras en sitio y realizar los ensayos de laboratorio.

\section{Agradecimientos}

A la Universidad Cooperativa de Colombia y sus docentes por suministrarnos información en sus bases de datos y por permitirnos presentar la primera etapa de esta investigación en el Primer Congreso Nacional de Vías, y a algunos grupos de estudiantes de ingeniería civil por colaborarnos con la traducción de artículos en inglés.

\section{Referencias}

[1] A. Buitrago, "Desastres de origen natural y antrópico que se presentaron en el Valle de Aburrá". Boletín de prensa Área Metropolitana del Valle de Aburrá, 2009.

[2] J. J. Restrepo, "Obducción y metamorfismo de ofiolitas triásicas en el flanco occidental del terreno Tahamí, cordillera Central de Colombia". Boletín de ciencias de la tierra, Bogotá, 2008.

[3] L.A. Capdevila. "Comportamiento tenso-deformacional de suelos loessicos contaminados con hidrocarburos", presentado en el IX Congreso Latinoamercano de Estudiantes de Ingenieria Civil coleic, Universidad Nacional de Córdoba, Valparaíso, Chile, 2013.

[4] E. R. Redolfi, "Suelos colapsables". Facultad de Ciencias Exactas, Físicas y Naturales Departamento de Construcciones Civiles, Bogotá, 2007.

[5] B. M. A. Rafie, R. Ziaie y M. Esmaeli, "Evaluación del potencial colapso del suelo: estudio caso de la estación del tren de Semman". Facultad de Ingeniería Civil, Imam Khomeini Universidad Internacional, Escuela de Ingeniería Ferroviaria de Irán, Universidad de Ciencia y Tecnología de Teherán, Irán, 2008. 
[6] A. Reginatto, "Predicción de la Susceptibilidad al Colapso”. II Reunión Argentina de Mecánica de Suelos e Ingeniería de Fundaciones. Cordoba, Argentina, 1970.
[7] J. da Silva, "Estudio de Colapsabilidad de arcilla porosa de Brasilia”. Tesis de Maestría, Universidad de Brasilia, Facultad de Tecnología, Departamento de Ingeniería Civil y Ambiental. Brasilia, Brasil, 2006. 